\title{
Raíces adventicias de Saxegothaea conspicua Lindl. (Podocarpaceae) en su etapa de senescencia en bosques adultos
}

\section{Adventitious roots of Saxegothaea conspicua Lindl. (Podocarpaceae) in its senescence stage in old-growth forests}

\author{
Mauricio J. Cano ${ }^{1}$, Roberto Godoy ${ }^{2} \&$ Daniel P. Soto $3,4^{*}$ \\ ${ }^{1}$ Institute of Biological Science, University of Edinburgh and Royal Botanic Garden of Edinburgh, 20A Inverleith Row, Edinburgh \\ EH9 3JU, Scotland, United Kingdom \\ ${ }^{2}$ Instituto de Ciencias Ambientales y Evolutivas, Universidad Austral de Chile, Isla Teja s/n, Valdivia, Chile. \\ ${ }^{3}$ Instituto de Bosques y Sociedad, Universidad Austral de Chile, Isla Teja s/n, Valdivia, Chile. \\ ${ }^{4}$ Department of Forest Ecosystems and Society, Oregon State University, Corvallis, OR 97331 USA. \\ *danielsoto@uach.cl
}

\begin{abstract}
We studied the anatomy of the adventitious structures that Saxegothaea conspicua Lindl. develops inside of the stem during its senescence stages in old-growth temperate forest; by scanning electronic microscopy, we evaluated the hypothesis whether those structures correspond to adventitious roots. The results confirm that the anatomical structures are similar to a common roots system at first development stages. We discuss the possible functional implications of these adventitious roots in extend the life of the individuals in forests.
\end{abstract}

Saxegothaea conspicua Lindl. es un árbol endémico de los bosques templados de Sudamérica, es monoico, tolerante a la sombra, y que puede alcanzar hasta $39 \mathrm{~m}$ de altura y 2,3 $\mathrm{m}$ de diámetro (Cano 2008). La especie se desarrolla en los bosques templados de Chile y Argentina, pero es escaso en el lado oriental de los Andes (Donoso et al. 2006). En Chile se encuentra entre el río Maule y la Región de Aisén (36$49^{\circ} \mathrm{S}$ ) (Donoso et al. 2006). La mayor dominancia se ubica principalmente bajo las condiciones de clima oceánico, sin embargo, es capaz de crecer en climas mediterráneos pero sólo en condiciones microclimáticas de mayor humedad. La especie tiende a crecer en suelos de alta fertilidad, húmedos pero bien drenados (Donoso et al. 2006).

En bosques adultos, $S$. conspicua presenta individuos senescentes en pie, con un fuste en avanzado nivel de descomposición interno, de una extensión variable (e.g. longitud y diámetro), del cual se originan internamente finas y largas estructuras vegetativas de carácter leñoso, las cuales se despliegan desde la copa entremezclado con restos del fuste leñoso biodegradado, hasta alcanzar finalmente el suelo para su fijación. Dicho fenómeno ha sido descrito como "raíces adventicias" (Fig. 1). El primer registro de estas "raíces adventicias" fue descrito por Veblen et al. (1981), en un estudio de dinámica de bosques. Estos autores infieren sobre las implicancias que le dan estas estructuras a $S$. conspicua sobre el desarrollo sucesional del bosque, concluyendo que tienen la capacidad de anclarse al sustrato, y que podrían cumplir un rol para perpetuar al individuo en el sitio (Veblen et al. 1981).

El presente trabajo tiene como objetivo describir la estructura que desarrolla $S$. conspicua en el interior del tronco y verificar si la morfo-anatomía corresponde a una "raíz adventicia". La hipótesis de este estudio es que las estructuras de carácter leñoso que desarrollan los individuos de $S$. conspicua en el interior de su fuste biodegradado, en su etapa de senescencia, corresponden a raíces adventicias.

El estudio se realizó en un rodal de bosque adulto dominado por árboles emergentes de Nothofagus dombeyi (Mirb.) Blume. donde el dosel principal estaba dominado por Laurelia sempervirens (Ruiz \& Pav.) Tul. y S. conspicua (Veblen et al. 1981, Donoso \& Lusk 2007). El rodal estudiado se ubica en el predio San Pablo de Tregua de la Universidad Austral de Chile, ubicado entre los $39^{\circ} 30^{\prime}$ a $39^{\circ} 38^{\prime} \mathrm{S}$ y los $72^{\circ} 02^{\prime}$ a $72^{\circ} 09^{\prime} \mathrm{O}$, y a altitudes entre los 550 a 850 m s.n.m. Mayores detalles sobre el clima, vegetación y el suelo del lugar de estudio son presentados por Donoso et al. (2007), Soto et al. (2009) y Oyarzún et al. (2011).

Para caracterizar la morfo-anatomía de la estructura estudiada en árboles de $S$. conspicua, se realizó un muestreo dirigido a individuos que presentaran un estado de degradación avanzada del fuste, y que manifestaran el fenómeno de crecimiento de estructuras de carácter leñoso en su interior (Fig. 1a y b), procurando que estas estructuras 
adventicias se encontraran en su primera etapa de desarrollo, debido a que permiten confirmar en forma inequívoca su funcionalidad, al comprobar la presencia de pelos radicales activos (vivos). Mayores detalles anatómicos en estadios temprano de raíces en coníferas chilenas en Godoy \& Mayr (1989).

El muestreo consistió en la realización de tres transectos de $100 \mathrm{~m}$ en tres microcuencas distintas. En cada transecto se seleccionaron 5 individuos que presentaran la mayor presencia de la estructura estudiada y por cada individuo seleccionado, se tomaron 15 muestras de las estructuras aéreas en su primera etapa de desarrollo. Las muestras se depositaron en frascos con fijador de F.A.A. (formalina, ácido acético y alcohol). Las muestras fueron seccionadas a una longitud aproximada de $2 \mathrm{~cm}$. Luego en laboratorio se siguió protocolos estándar para el procesamiento de muestras para microscopia electrónica de barrido (Godoy \& Mayr 1989). Se escogieron al azar 5 muestras por individuo para comprobar el patrón morfo-anatómico, las que fueron dimensionadas aproximadamente a una longitud de 3 a 5 $\mathrm{mm}$, para depositar en envases de vidrio y fijadas en alcohol $50 \%$. Posteriormente se hicieron 10 cortes transversales y 10 longitudinales con un micrótomo (Godoy \& Mayr 1989, Steubing et al. 2002). El microscopio electrónico de barrido utilizado fue un Leitz Electron Optic-LEO, Modelo LEO 420, implementado con microanalizador de Rayos X (Oxford Instrument inc.).

Por medio de las imágenes de microscopía, se pudo verificar que la estructura de carácter leñoso que desarrolla $S$. conspicua en el interior del fuste biodegradado corresponde
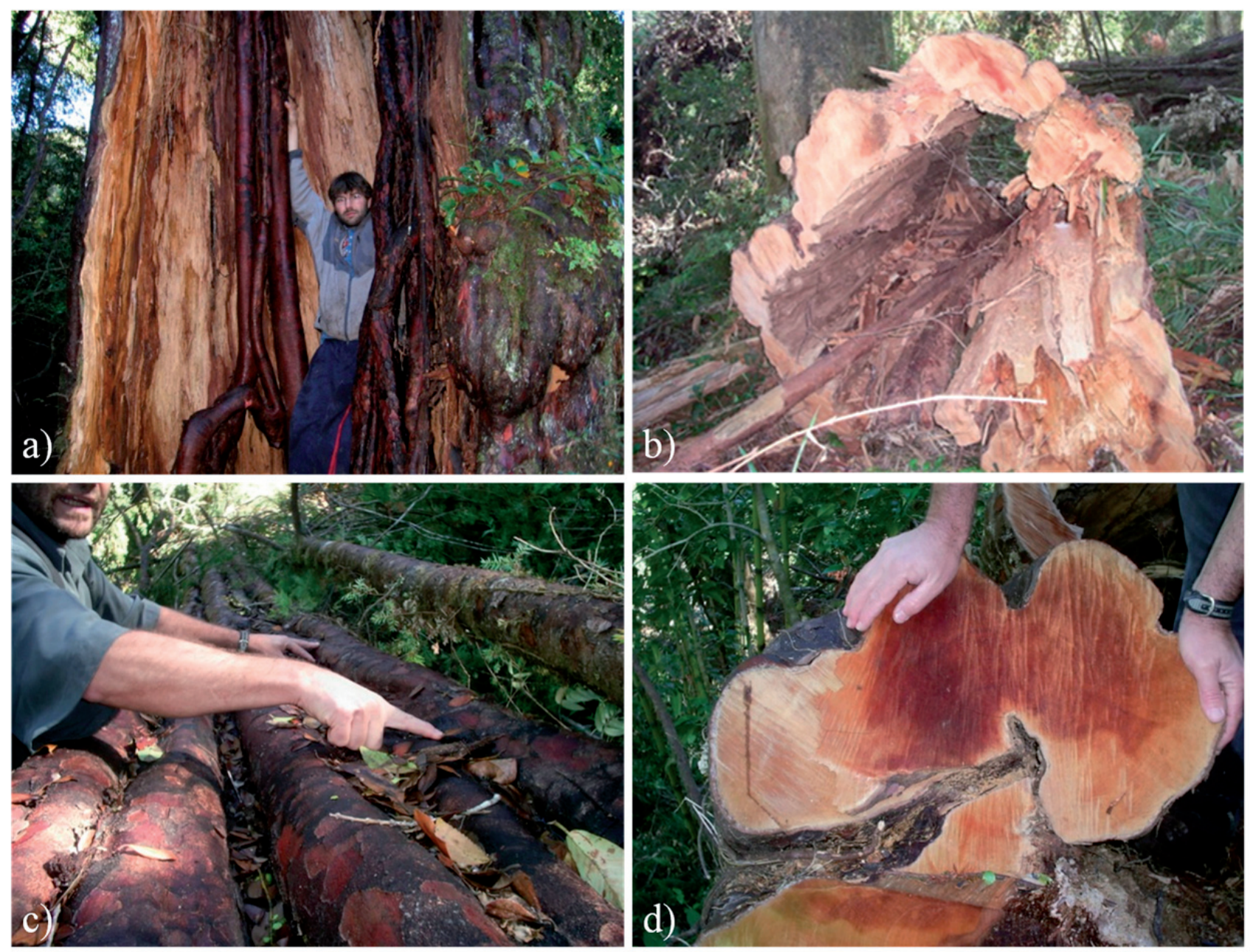

Figura 1. Individuos de Saxegothaea conspicua. a) Estructuras de carácter leñoso (raíz adventicia), en avanzado estado de desarrollo. b) Fuste con un avanzado estado de degradación, con presencia de "raíces adventicias". c) Vista longitudinal de raíces adventicias mostrando el fenómeno de anastomosis, d) vista transversal de raíces adventicias mostrando fenómeno de anastomosis.

FIGURE 1. Saxegothaea conspicua individuals. a) Woody structures (adventitious roots), in advanced stages of development. b) Stem with an advanced state of decay, with presence of "adventitious roots". c) Longitudinal view of adventitious roots showing the "anastomosis" phenomenon. d) Transversal view of the adventitious roots showing the anastomosis phenomenon. 
a una raíz adventicia (Fig. 2a). Las estructuras estudiadas presentan una morfología típica de una raíz de coníferas (sensu Godoy \& Mayr 1989), con clara endodermis, cortex (Fig. 2a), pelos radicales (Fig. 2b y c) y abundantes nódulos radicales, correspondiendo a raíces modificadas (Fig. $2 \mathrm{~d}$, e y f). Cada nódulo presentó un tamaño aproximado de 0,5 $\mathrm{mm}$ de diámetro promedio, con una distribución de forma paralela en la superficie radical (Fig. 2d), característica típica de S. conspicua (Godoy \& Mayr 1989). Claramente la presencia de abundantes pelos radicales turgentes en los nódulos radicales fue el factor determinante para discriminar si corresponde a una raíz (Fig. 2 c). En la sección transversal de la raíz se pudo apreciar que está constituida por células parenquimáticas, de paredes delgadas, y prácticamente sin espacios intercelulares (Fig. 2a y b).
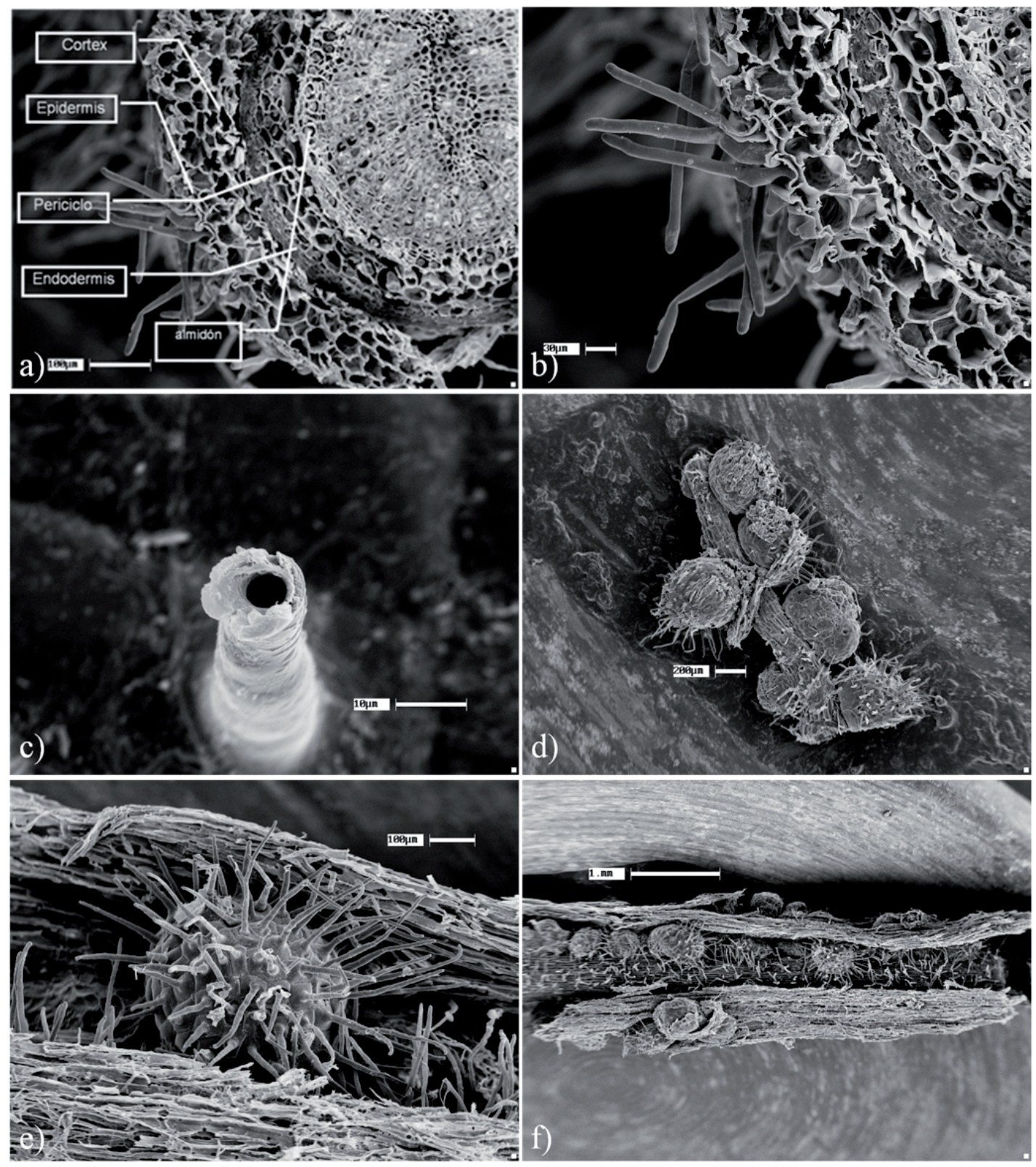

FIGURA 2. Imágenes de microscopía electrónica de barrido para el material de estudio radical en su primera etapa de desarrollo. a) Corte transversal de raíz adventicia. b) Corte transversal enfatizando distribución de pelos radicales (PR). c) Detalle de pelo radical. d) Distribución paralela de nódulos radicales. e y f) Nódulos radicales.

FiguRE 2. Scanning electron microscopy images of the study material at the first development stage. a) Cross-section of adventitious roots, b) Cross-section emphasizing distribution of roots hairs (PR). c) Detail of root hair. d) Parallel distribution of root nodules. e) and f) root nodules. 
Las eventuales implicaciones sucesionales de estas raíces han sido mencionadas por diversos estudios (Veblen et al. 1981, Donoso 1993). En nuestras observaciones de terreno fue posible encontrar individuos de $S$. conspicua con avanzado estado de descomposición del fuste, donde se desarrollan raíces adventicias que se despliegan hasta llegar al suelo, otorgando a posteriori una función mecánica de anclaje, y posterior desarrollo leñoso, que en varios estadios le permitirían el sustento y persistencia del individuo en la comunidad forestal (Veblen et al. 1981, Cano 2008), lo que resultaría en una estrategia de sobrevivencia efectiva para la especie (Fig. 1a y b). Adicionalmente, observaciones de terreno permiten inferir que estas raíces adventicias, además de desarrollarse hasta anclarse exitosamente en el sustrato, poseen la capacidad de unirse entre ellas en la parte aérea por medio de una fusión, que hemos denominado "anastomosis", generando un nuevo fuste, con un aumento considerable de la envergadura diametral, otorgándole una mayor estabilidad a la función mecánica de anclaje al individuo (Fig. 1c y d).

Este fenómeno consolida a $S$. conspicua como una especie única dentro de las podocarpáceas, siendo caracterizada y separada morfológica y evolutivamente del grupo (Mill \& Stark-Shilling 2009). Estudios más a fondo se requieren para analizar las implicancias ecológicas, evolutivas y funcionales que puede generar este fenómeno en los bosques templados del Centro-Sur de Chile.

\section{AGRADECIMIENTOS}

R.G. agradece a Fondecyt 1110331 y a Ricardo Silva en Microscopía. Se agradece las sugerencias de dos revisores anónimos y de la editora A. Marticorena.

\section{DEDICATORIA}

A Darian Stark, incansable divulgador de la flora de Chile y creador de la página web www.florachilena.cl.

\section{REFERENCIAS}

Cano, M.J. 2008. Estrategia de Saxegothaea conspicua para perpetuarse en el sitio. Tesis Pre-grado, Facultad de Ciencias Forestales y Recursos Naturales, Universidad Austral de Chile. 39 pp.

Donoso, C. 1993. Bosques Templados de Chile y Argentina. Variación, estructura y dinámica. 1 $1^{\mathrm{a}}$ Edición. Editorial Universitaria. Santiago, Chile. 483 pp.

Donoso, C., B. Escobar, P. Donoso, F. Ultreras \& A. Zúñiga. 2006. Saxegothaea conspicua. En: C. Donoso (ed.), Las especies arbóreas de Chile y Argentina, autoecología. Editorial Marisa Cuneo. 116-125 pp.

Donoso, P.J. \& C.H. LusK. 2007. Differential effects of emergent Nothofagus dombeyi on growth and basal area of canopy species in an old-growth temperate rainforest. Journal of Vegetation Science 18: 675-684.

Donoso, P.J., V. Gerding, D. Uteau, D.P. Soto, O.Thiers \& C. Donoso. 2007. Efecto de fertilización y cobertura de malezas en el crecimiento inicial y la mortalidad de una plantación de Nothofagus dombeyi en la Cordillera de Los Andes. Bosque 28(3): 249-255.

Godoy, R. \& R. Mayr. 1989. Caracterización morfológica de micorrizas vesículo-arbusculares en coníferas endémicas del sur de Chile. Bosque 10(2): 89-98.

Mill, R.R. \& D. Stark-Shilling. 2009. Cuticular micromorphology of Saxegothaea Lindl. (Podocarpaceae). Botanical Journal of the Linnean Society 159(1): 58-67.

Oyarzún, C., R. Godoy, J. Staelens, P.J. Donoso \& N. Verhoest. 2011. Rainfall partitioning in temperate rainforest in the Andes of South-Central Chile is related to forest structure and tree characteristics. Hydrological Processes 25(4): 623-633.

Soto, D.P., P.J. Donoso, D. Uteau \& A. ZúÑIga-Feest. 2009. Environmental factors affect the spatial arrangement of survival and damage of outplanted Nothofagus dombeyi seedlings in the Chilean Andes. Interciencia 34: 100-105.

Steubing, L., R. Godoy \& M. Alberdi. 2002. Métodos de ecología vegetal. Editorial Universitaria.Santiago, Chile. 345 pp.

Veblen, T.T., C. Donoso, F.M. Schlegel \& B. Escobar. 1981. Forest dynamics in south-central Chile. Journal of Biogeography 8: 211-247.

Recibido: 08.03.13

Aceptado: 26.11.13 